Check for updates

Cite this: RSC Adv., 2018, 8, 27382

\title{
Nitroxide radical-modified CuS nanoparticles for CT/MRI imaging-guided NIR-II laser responsive photothermal cancer therapy
}

\author{
Chengwan Xia, $\dagger^{a}$ Diya Xie, $\dagger^{a}$ Lang Xiong, ${ }^{a}$ Qian Zhang, ${ }^{a}$ Yang Wang, ${ }^{a}$ Zezheng Wang, ${ }^{a}$ \\ Yuxin Wang, ${ }^{\star a}$ Bin Li $\mathbb{D}^{\star a}$ and Chao Zhang*ab
}

Received 26th May 2018

Accepted 14th July 2018

DOI: $10.1039 / c 8 \mathrm{ra04501a}$

rsc.li/rsc-advances

\begin{abstract}
Herein, we reported nitroxide radical-modified CuS nanoparticles (CUS-NO` NPs), and they exhibited a typical absorption peak at $1182 \mathrm{~nm}$. Due to such a long wavelength absorbance, CuS-NO* NPs exhibited excellent therapeutic outcome and low damage to normal tissues. Besides, we simultaneously achieved CuS-NO• NPs for MRI and CT dual-modal imaging, which successfully provided a new strategy for imaging-guided tumor treatment, thus increasing potential clinical applications for cancer treatment.
\end{abstract}

\section{Introduction}

Many evidences have indicated that traditional cancer treatments are failing to eradicate tumors. There are two major reasons for this. On the one hand, oncotherapy is seriously influenced by tumor environment, which assists the invasion and metastasis of tumor cells and leads to cancer recurrence. ${ }^{1-4}$ On the other hand, traditional cancer treatments such as surgery, chemotherapy, and radiation result in indistinguishable small lesions, extreme drug resistance, and carcinogenesis in normal tissues. ${ }^{5,6}$ In this regard, it is essential to find new avenues for cancer treatment.

Photothermal therapy (PTT) has attracted tremendous attention owing to its noninvasive nature and high photothermal conversion efficiency. ${ }^{7-18}$ Ideal PTT agents are capable of converting absorbed optical energy into heat. Good optical energy absorption is a prerequisite for such materials. Based on this, many PTT agents based on noblemetals, semiconductors, carbon, and polymers have been developed. ${ }^{19-26}$ However, most of these PTT agents display short wavelength absorbance, thus limiting tissue penetration depths and maximum permissible exposure (MPE). ${ }^{27-29}$ NIR-I ranging from $650 \mathrm{~nm}$ to $950 \mathrm{~nm}$ and NIR-II ranging from $1000 \mathrm{~nm}$ to $1350 \mathrm{~nm}$ are two NIR biological windows. ${ }^{30}$ Comparatively speaking, although the NIR-I window has been more widely researched, the NIR-II window permits deeper tissue penetration depths and higher MPE of lasers. ${ }^{31}$ Unfortunately, studies concerning PTT treatment in the NIR-

${ }^{a}$ Nanjing Stomatological Hospital, Medical School of Nanjing University, Nanjing, Jiangsu 210093, P. R. China. E-mail: 904489973@qq.com; 1libin@163.com; 1446980166@qq.com

${ }^{b}$ Collaborative Innovation Center of Chemistry for Life Sciences, College of Engineering and Applied Sciences, Nanjing University, Nanjing, Jiangsu, 210093, China

$\dagger$ These authors contributed equally to this work.
II region have rarely been reported. Therefore, the synthesis of NIR-II laser-responsive PTT agents capable of achieving deeper tissue penetration depths and higher MPE is vital but remains challenging.

Herein, we successfully synthesized nitroxide radicalmodified CuS nanoparticles (CuS-NO' NPs), which have a typical absorption peak at $1182 \mathrm{~nm}$. The absorption peak was much higher than those previously reported. ${ }^{32}$ Based on this, a $1177 \mathrm{~nm}$ laser was employed to investigate the photothermal conversion efficiency in PTT treatment. Due to such a long wavelength absorbance, CuS-NO NPs exhibited excellent therapeutic outcome and low damage to normal tissues, which were demonstrated by in vitro and in vivo tests. Besides, both the CuS nanoparticles (CuS NPs) and NO' radicals were stable and separately contributed to enhanced $\mathrm{CT}$ and $\mathrm{T}_{1}$-weighted MR imaging, respectively. ${ }^{33-37}$ In principle, CT imaging suffers from low spatial resolution, whereas MR imaging has no response to calcification. In this system, CuS-NO NPs were simultaneously achieved for MRI and CT dual-modal imaging, thus successfully compensating for the low spatial resolution rendered by single CT imaging and the negative response to calcification of single MR imaging. Although some nanoparticles can be used for CT/ MR dual mode imaging, they are usually composed of multicomponent metals, thereby leading to heavy-metal toxicities. ${ }^{38}$ We believe these tailor-made CuS-NO ${ }^{*}$ NPs will provide a new strategy for CT/MR imaging-guided NIR-II laser-responsive PTT treatment and increase the possible clinical applications in cancer treatment.

\section{Experimental section}

Materials and animals

$\mathrm{CuCl}_{2} \cdot 2 \mathrm{H}_{2} \mathrm{O}$, sodium hydroxide $(\mathrm{NaOH})$, and thioacetamide were purchased from Aladdin (Shanghai, China). SH-PEG- 
$\mathrm{COOH}\left(M_{\mathrm{w}} 20000 \mathrm{Da}\right)$ was bought from J\&K Chemicals (Beijing, China). $\quad N$-(3-Dimethylaminopropyl)- $N^{\prime}$-ethylcarbodiimide hydrochloride (EDC, Bioxtra), N-hydroxysuccinamide (NHS, 98.0\%), and phosphate-buffered saline (PBS, pH 7.4) were obtained from Jinpan Biotech (Nantong, China). Human umbilical vein endothelial cells (HUVECs) and human cervical carcinoma (HeLa) cell line were purchased from Servicebio Company (Wuhan, China). Nude mice (female, 4-6 weeks) were bought from Comparative Medicine Centre of Yangzhou University. All animal experiments were approved by the Care Committee of Nanjing University (including guidelines for animal care and use and guidelines for euthanasia of mice, Protocol \#20170322-016).

\section{Preparation of CuS-NO NPs}

CuS-NO` NPs were synthesized as illustrated in Scheme 1. First, an aqueous solution $(50 \mathrm{~mL})$ of SH-PEG-COOH $(0.1$ $\mathrm{mmol})$ was mixed with an aqueous solution $(50 \mathrm{~mL})$ of $\mathrm{CuCl}_{2} \cdot 2 \mathrm{H}_{2} \mathrm{O}(0.1 \mathrm{mmol})$ and reacted for $1 \mathrm{~h}$. Afterward, an aqueous solution $(1 \mathrm{~mL})$ of $\mathrm{NaOH}(0.5 \mathrm{mmol})$ was added dropwise to adjust the solution $\mathrm{pH}$ to 9 . Then, the solution was transferred to a three-necked flask, and an aqueous solution (2 $\mathrm{mL})$ of thioacetamide $(0.1 \mathrm{mmol})$ was added. The reaction was carried out under constant stirring at $60^{\circ} \mathrm{C}$ for $1.5 \mathrm{~h}$, and the process was protected by $\mathrm{N}_{2}$ atmosphere. Conjugation of $\mathrm{NO}^{\circ}$ was performed by activating the carboxyl groups of CuS NPs. Briefly, EDC (3 mg), sulfo-NHS (1.8 mg) and 4-amino-2,2,6,6tetramethylpiperidinooxy free radicals $(1 \mathrm{mg})$ were added to CuS NP suspension (10 mL DI water, $0.1 \mathrm{mg} \mathrm{mL}^{-1}$ ). Conjugation proceeded for $4 \mathrm{~h}$ at room temperature under continuous stirring. Afterwards, the resultant $\mathrm{CuS}^{\mathrm{NO}}{ }^{*} \mathrm{NPs}$ were harvested by centrifuging three times and washing with PBS to remove non-specifically absorbed $\mathrm{NO}^{\circ}$ and excess reactants. Purified CuS-NO ${ }^{\circ}$ NPs were re-suspended in PBS and stored at $4{ }^{\circ} \mathrm{C}$ before use.
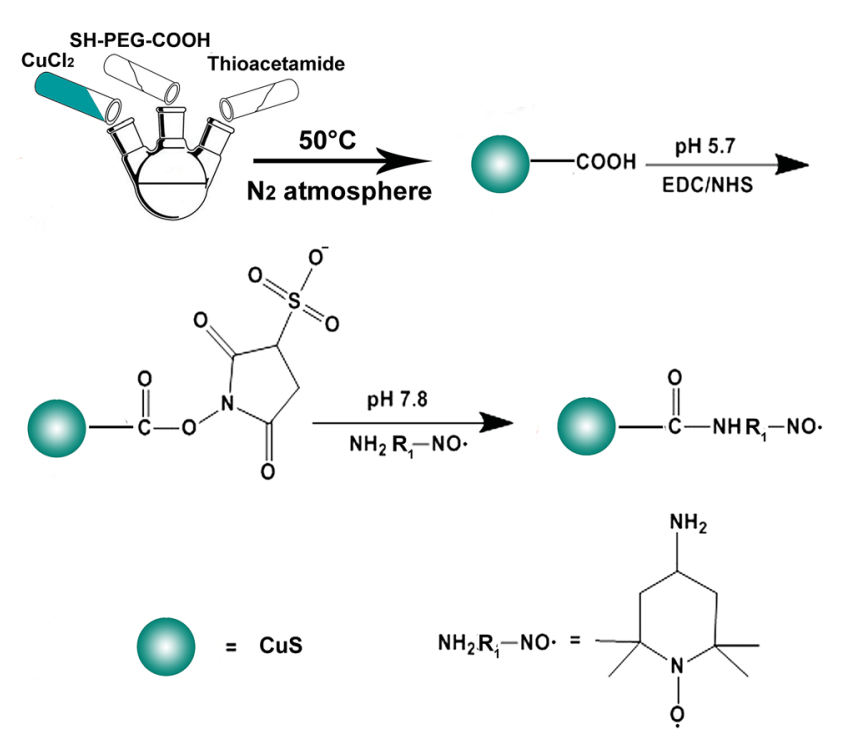

Scheme 1 Synthesis of CuS-NO•NPs.

\section{Characterization}

The morphologies of the obtained CuS NPs and CuS-NO NPs were observed by transmission electron microscopy (TEM, JEOL), and UV-vis absorption spectra were recorded on a spectrophotometer (UV3100, Shimadzu, Japan). Size distribution was confirmed by using a dynamic light scattering (DLS) instrument (Brookhaven, BI-9000AT, USA). The signal of NO ${ }^{\circ}$ in CuS-NO ${ }^{*}$ NPs was tested by electron paramagnetic resonance (EPR) experiments. To study photothermal effects, a $1177 \mathrm{~nm}$ diode laser (LE-LS-1177-3000TFC-D, LaserGlow Technologies, Toronto, Canada) was used. The photothermal conversion efficiency $(\eta)$ was derived from the following formula ${ }^{10,18}(w$ represents laser power, $0.5 \mathrm{~W} ; m$ represents mass of CuS-NO ${ }^{*} \mathrm{NP}$ solution, $1.1 \times 10^{-3} \mathrm{~kg} ; t$ represents laser irradiation time, $600 \mathrm{~s} ; c$ represents specific heat capacity of CuS-NO NP solu-

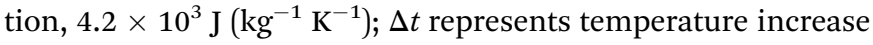
of PEG-DA-CuS NP solution):

$$
(\%)=\frac{c m \Delta t}{w t} \times 100 \%=\frac{4.2 \times 10^{3} \times 1.1 \times 10^{-3} \Delta t}{0.5 \times 600} \times 100 \%
$$

\section{MTT assay}

An MTT test was used to evaluate the effect of CuS-NO ${ }^{*}$ NPs on cell viability. Briefly, HUVECs were inoculated in 96-well plates $\left(3 \times 10^{3}\right.$ cells per well). After $12 \mathrm{~h}$, the culture medium supernatant was discarded. Then, the cells were washed with PBS (pH 7.4) and co-cultured with different concentrations of CuS-NO NPs for $24 \mathrm{~h}$. A $20 \mu \mathrm{L}$ volume per well of MTT was added into 96well plates. After $4 \mathrm{~h}$, DMSO was used to dissolve the blue formazan crystals for $0.5 \mathrm{~h}$. The absorbance of 96-well plates was obtained at $490 \mathrm{~nm}$ by an ELISA plate reader (BIO-RAD Inc., USA).

\section{In vivo PET imaging}

In vivo PET imaging was carried out to estimate the hypoxic level in HeLa-xenograft-bearing mice after NIR-II laser-responsive PTT treatment rendered by CuS-NO ${ }^{\circ}$ NPs. Each mouse was intravenously administrated with $100 \mu \mathrm{L}$ saline containing ${ }^{18} \mathrm{~F}$ MISO $(75 \mu \mathrm{Ci})$ before treatment. Mice were scanned by the Inveon PET/CT system (Siemens, GER) in a micro-PET scan $1 \mathrm{~h}$ post administration.

\section{CT imaging assay}

A clinical CT Gemstone Spectral 64-Detector CT (Discovery CT 750HD, GE Amersham Healthcare System, Milwaukee, WI) was used for all CT scans. For in vitro CT imaging, different concentrations of CuS-NO ${ }^{*}$ PP solution $(0,1,3,5$, and $7 \mathrm{mg}$ $\mathrm{mL}^{-1}$ ) were added to a 6-well plate. For in vivo CT imaging, HeLa-xenograft-bearing mice received intratumoral injection of CuS-NO NPs or iodixanol ( $\left.4 \mathrm{mg} \mathrm{kg}^{-1}\right)$ solution, and the scans were conducted $1 \mathrm{~h}$ post intratumoral injection. 


\section{MR imaging study}

A 7.0 T small animal MR scanner (Bruker PharmaScan, Germany) was used for all MR scans. A turbo spin echo sequence (parameters: field of view $=150 \times 150 \mathrm{~mm}$; repetition time $=$ 400-675 ms; echo time $=18 \mathrm{~ms}$; slice thickness $=1 \mathrm{~mm}$; slice gap $=$ default; matrix $=188 \times 151$; number of signals averaged $=2$ ) were investigated by TEM. As shoaging. Total scan duration was set at $48 \mathrm{~s}$. A turbo spin echo sequence was used for in vivo $\mathrm{T}_{1}$-weighted MR imaging with a field of view of $3.0 \times 3.0 \mathrm{~cm}$, twelve axial slices with a slice thickness of $1 \mathrm{~mm}$, matrix size of $256 \times 256$, repetition time of $500 \mathrm{~ms}$, and total scan time of $120 \mathrm{~s}$.

\section{Infrared thermal imaging study}

An infrared thermographic camera (ThermaCAM SC3000, FLIR, USA) was employed to monitor and record the change of temperature of HeLa-xenograft-bearing mice intratumorally injected with PBS or CuS-NO ${ }^{\bullet}$ NPs $\left(100 \mu \mathrm{L}, 1 \mathrm{mg} \mathrm{mL}^{-1}\right)$ solution and exposed to a $1177 \mathrm{~nm}$ laser. Irradiation was carried out $1 \mathrm{~h}$ after injection with output power density of $0.5 \mathrm{~W} \mathrm{~cm} \mathrm{~cm}^{-2}$ and irradiation time of $10 \mathrm{~min}$.

\section{In vitro antitumor effect}

The Annexin-V-FITC/propidium iodide (PI) staining method was employed to assess in vitro PTT rendered by CuS-NO ${ }^{*}$ NPs. Briefly, HeLa cells were seeded into 96 -well plates $\left(1 \times 10^{4}\right.$ cells and $100 \mathrm{~mL}$ medium per well) and incubated for one day. Then, the cells were washed twice with PBS, followed by incubation with CuS-NO ${ }^{*}$ NPs. Afterward, the cells were cultured with fresh culture medium at $37^{\circ} \mathrm{C}$ for $4 \mathrm{~h}$. Finally, the cells were collected and resuspended in a binding buffer $(500 \mu \mathrm{L})$. Annexin V-FITC and PI were added following the manufacturer's guidelines. After incubation in darkness at room temperature for $20 \mathrm{~min}$, the samples were analysed using flow-cytometry (FACSVerse, BD Bioscience) and the relevant FlowJo software.

\section{In vivo antitumor effect}

HeLa-xenograft-bearing mice, with an average tumor size of 500 $\mathrm{mm}^{3}$, were employed to assess in vivo antineoplastic efficiency. The mice were randomized into four different groups (intratumoral injection of saline $\left(4 \mathrm{mg} \mathrm{kg}^{-1}\right)$, intratumoral injection of CuS-NO ${ }^{\cdot}$ NPs ( $4 \mathrm{mg} \mathrm{kg}{ }^{-1}$ ) alone, intratumoral injection of CuS NPs (4 mg kg${ }^{-1}$ ) plus $1177 \mathrm{~nm}$ laser, and intratumoral injection of CuS-NO NPs ( $4 \mathrm{mg} \mathrm{kg}^{-1}$ ) plus $1177 \mathrm{~nm}$ laser). Tumor size was evaluated by monitoring and recording the volume. Survival and body weight of each mouse were also monitored and recorded.

\section{Pathology analysis}

Organs (lung, heart, spleen, liver, and kidney) were collected from the mice on day 7, day 15 and day 30 after NIR-II laser response PTT treatment rendered by CuS-NO` NPs or from untreated normal mice to observe histological changes. These collected organs were immobilized with $4 \%$ paraformaldehyde and then embedded with paraffin. Finally, representative images were obtained using a light microscope after the sections received hematoxylin and eosin (H\&E) staining.

\section{Hematology and biochemical assay}

Hematology and biochemical assays were conducted by collecting mouse blood on day 7, day 15 and day 30 after NIR-II laser-responsive PTT treatment rendered by CuS-NO` NPs or from untreated normal mice. Peripheral blood lymphocytes (LYM), blood urea nitrogen (BUN), monocytes (MON), platelets (PLT), behavioral approach system (BAS), alanine aminotransferase (ALT), aminopherase (AST), red blood cells (RBC), serum globulin (GLB), eosinophils (EOS), alkaline phosphatase (ALP), and creatinine $(\mathrm{Cr})$ were all monitored to assess biocompatibility and in vivo toxicity of CuS-NO` NPs.

\section{Results and discussion}

\section{Characterisation}

The morphologies of the as-obtained CuS NPs and CuS-NO• NPs were investigated by TEM. As shown in Fig. $1 \mathrm{~A}$ and $\mathrm{B}$, the addition of $\mathrm{NO}^{*}$ did not change the size and morphology of CuS NPs; both CuS NPs and CuS-NO` NPs exhibited a morphology of homogeneously dispersed spheres and a mean diameter of about $10.8 \mathrm{~nm}$. Furthermore, there was a slight increase in the hydrodynamic diameter after modification of NO` on CuS NPs, as measured by dynamic light scattering (Fig. 1C). The presence of $\mathrm{NO}^{\circ}$, featuring a triplet-curve in the electron paramagnetic resonance (EPR) spectrum, was confirmed on the surface of CuS
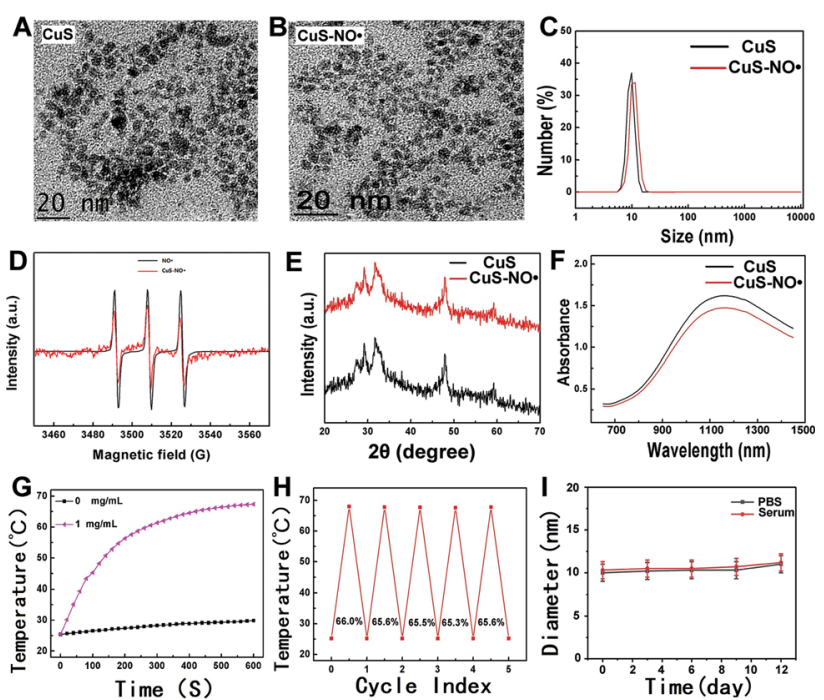

Fig. 1 Characterization of CUS-NO* NPs. (A) TEM of CuS NPs. (B) TEM of CuS-NO* NPs. (C) DLS of CuS-NO ${ }^{*}$ NPs. (D) EPR of NO and CuSNO ${ }^{\circ}$ NPs. (E) XRD of CuS NPs and CUS-NO NPs. (F) UV-vis spectra of CuS NPs and CuS-NO* NPs. (G) Temperature curves of CuS-NO* NPs irradiated with a $1177 \mathrm{~nm}$ laser $\left(0.5 \mathrm{~W} \mathrm{~cm}^{-2}\right)\left(0 \mathrm{mg} \mathrm{mL}^{-1}, 1.0 \mathrm{mg} \mathrm{mL}^{-1}\right)$ as a function of time $(0-600 \mathrm{~s})$. (H) Temperature elevation of CuS$\mathrm{NO} \cdot \mathrm{NP}$ solution $(1.1 \mathrm{~g}, 1 \mathrm{~mL})$ treated with five cycles of $1177 \mathrm{~nm}$ laser irradiation $\left(0.5 \mathrm{~W} \mathrm{~cm}^{-2}, 10 \mathrm{~min}\right)$ and corresponding natural cooling (0.5 h). (I) Stability investigation of CuS-NO* NPs in PBS or serum via hydrodynamic diameter. 
NPs (Fig. 1D). Fig. 1E shows the XRD pattern of CuS-NO` NPs, which is in agreement with the powder diffraction pattern of CuS (JCPDS no. 06-0464). High absorption in the NIR-II region is the prerequisite for PTT agents for use in NIR-II laser-responsive PTT treatment. As expected, both CuS NPs and CuS-NO` NPs showed significant absorption intensities at about 1100$1300 \mathrm{~nm}$ in the UV spectra, and a maximum absorption peak at $1182 \mathrm{~nm}$ was displayed (Fig. 1F). For this reason, a $1177 \mathrm{~nm}$ laser with laser power of $0.5 \mathrm{~W} \mathrm{~cm}^{-2}$ was used to irradiate the CuS-NO ${ }^{*} \mathrm{NP}$ solution $\left(1 \mathrm{~mL}, 1 \mathrm{mg} \mathrm{mL}^{-1}\right)$, and an infrared thermographic camera was employed to monitor and record temperature elevation. As exhibited in Fig. 1G, we could observe a sharp rise in temperature in the $\mathrm{CuS}-\mathrm{NO}^{\circ}$ group, and the temperature reached near $70{ }^{\circ} \mathrm{C}$ after $10 \mathrm{~min}$ irradiation, demonstrating the highly efficient NIR-II laser energy-heat conversion capacity of CuS-NO ${ }^{*}$ NPs. Fig. $1 \mathrm{H}$ shows the conversion efficiency of CuS-NO ${ }^{*} \mathrm{NPs}$, and the values were near $66 \%$ without clear change in the five cycles of laser irradiation, indicating $\mathrm{CuS}-\mathrm{NO}^{*}$ NPs could play an active role in NIR-II laserresponsive PTT treatment. It is worth mentioning that these CuS-NO NPs did not show any change in hydrodynamic diameter in PBS or acidic PBS, confirming their potential to be applied in the body.

\section{MTT assay}

The cytotoxicity of CuS-NO' was evaluated via MTT analysis (Fig. 2A and B). The cells safely tolerated CuS-NO' below a concentration of $1 \mathrm{mg} \mathrm{mL}{ }^{-1}$, as indicated by negligible loss of viability $(<4 \%$, Fig. $2 \mathrm{~A})$. Even when the nanoparticles were introduced in concentration as high as $5 \mathrm{mg} \mathrm{mL}^{-1}$, over $85 \%$ viability could still be reached (Fig. 2A), further suggesting that

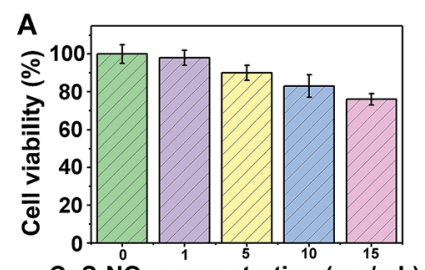
CuS-NO• concentration $(\mathrm{mg} / \mathrm{mL})$
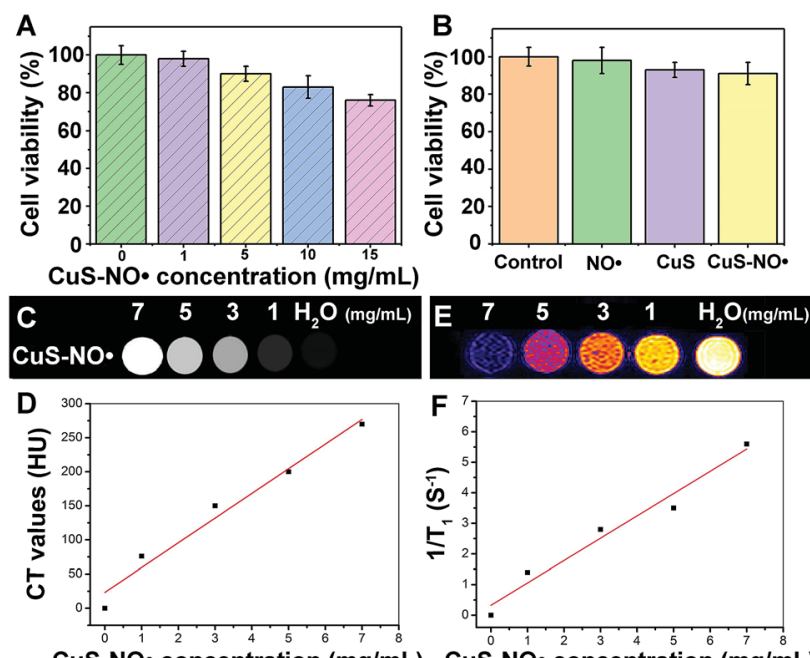

CuS-NO• concentration $(\mathrm{mg} / \mathrm{mL})$

CuS-NO• concentration $\left(\mathrm{mg} / \mathrm{mL}^{2}\right)$

Fig. 2 (A) MTT for different concentrations of CuS-NO* NPs. (B) MTT for different groups of CuS-NO ${ }^{*} \mathrm{NPs}$, CuS NPs, NO and control at the concentration of $5 \mathrm{mg} \mathrm{mL}^{-1}$. (C) CT of CuS-NO NPs in vitro. (D) Hounsfield unit (HU) values for different concentrations of CuS-NO' NPs. (E) $\mathrm{T}_{1}$-weighted phantom images of CuS-NO* NPs with different concentrations at 7.0 T. (F) The linear fitting of longitudinal relaxation rates at $7.0 \mathrm{~T}$ versus concentrations of CuS-NO NPs.
CuS-NO ${ }^{*}$ NPs featured good biocompatibility and were suitable for in vivo applications. Furthermore, in each group (CuS-NO NPs, CuS NPs or NO'), there was no difference in MTT assay results after $24 \mathrm{~h}$ incubation (Fig. 2B) at $5 \mathrm{mg} \mathrm{mL}^{-1}$. Based on this, a concentration of $5 \mathrm{mg} \mathrm{mL} \mathrm{mL}^{-1} \mathrm{CuS}-\mathrm{NO}^{*}$ NPs was adopted in the following experiments.

\section{In vitro $\mathrm{CT}$ and MRI studies}

CuS NPs have been reported for use in CT imaging. ${ }^{39}$ As such, CT scans of CuS-NO ${ }^{*}$ NPs were also performed to evaluate the feasibility of $\mathrm{CuS}-\mathrm{NO}^{*} \mathrm{NPs}$ for use as CT contrast agents. In vitro CT imaging is shown in Fig. 2C. It was clear that CuS-NO ${ }^{*}$ NPs could be used for CT imaging as they showed comparably bright images (Fig. 2C). CT contrast brightness and Hounsfield unit (HU) values of CuS-NO' NPs increased linearly with the concentration of $\mathrm{CuS}^{-\mathrm{NO}}{ }^{\circ} \mathrm{NPs}$ (Fig. 2D). At present, multimodal imaging technology has attracted widespread attention in biomedical applications. In particular, the combination of MRI and CT is a prospect for the development of more sensitive, precise biological imaging systems and imaging guidance therapy.

Owing to its high ${ }^{1} \mathrm{H}$ water relaxivities $\left(r_{1}\right)$, superparamagnetic performance, and long in vivo circulation, ${ }^{35-37}$ $\mathrm{NO}^{\bullet}$ has been widely investigated as a $\mathrm{T}_{1}$ MRI contrast agent. We hypothesized that $\mathrm{CuS}^{-\mathrm{NO}^{\circ}}$ NPs might also be used for MR imaging. Therefore, different concentrations of $\mathrm{CuS}-\mathrm{NO}^{\circ} \mathrm{NP}$ aqueous solutions $\left(0,1,3,5\right.$, and $\left.7 \mathrm{mg} \mathrm{mL}^{-1}\right)$ were studied for in vitro MRI. Fig. $2 \mathrm{E}$ and $\mathrm{F}$ show that CuS-NO ${ }^{*}$ NPs had comparably bright $\mathrm{T}_{1}$-weighted phantom images, and the brightness of $\mathrm{T}_{1}$-weighted phantom images and the longitudinal relaxivity $\left(r_{1}\right)$ value both increased linearly with the concentration of CuSNO^ NPs, indicating superior MRI function.

\section{In vivo $\mathrm{CT}$ and MR imaging}

To assess the potential applications of CuS-NO ${ }^{*}$ NPs for in vivo CT imaging, HeLa-tumor-bearing mice were intratumorally injected with CuS-NO` NPs or iodixanol. Significant CT signal was seen for the tumor injected with $\mathrm{CuS}-\mathrm{NO}^{*} \mathrm{NPs}$, whereas no CT signal was observed for the tumor injected with iodixanol (Fig. 3A). This is reasonable because iodixanol as a small molecule has poor retention in tumors. ${ }^{\mathbf{4 0}-42}$ These results demonstrated that $\mathrm{CuS}^{-\mathrm{NO}}{ }^{*}$ NPs hold promise as CT contrast agents to locate tumor sites. Encouraged by the superior CT imaging capacity in vivo, the feasibility of CuS-NO ${ }^{*}$ NPs for in vivo MRI was examined. The mice were anaesthetized, intratumorally injected with CuS-NO ${ }^{*} \mathrm{NP}\left(4 \mathrm{mg} \mathrm{kg}^{-1}\right)$ solution, and then scanned with the MRI system at $30 \mathrm{~min}, 120 \mathrm{~min}$, and $300 \mathrm{~min}$. As expected, enhanced $\mathrm{T}_{1}$-weighted images were continuously observed over time via intratumoral injection of CuS-NO` NPs (Fig. 3B). Taken together, all data suggested that CuS-NO ${ }^{*}$ NPs possessed a long time window in the CT/MRIguided tumour treatment.

\section{Infrared (IR) thermal imaging}

HeLa-xenograft-bearing mice were treated with intratumoral injection of $\mathrm{PBS}$ or $\mathrm{CuS}-\mathrm{NO}^{*} \mathrm{NPs}$ and directly exposed to 

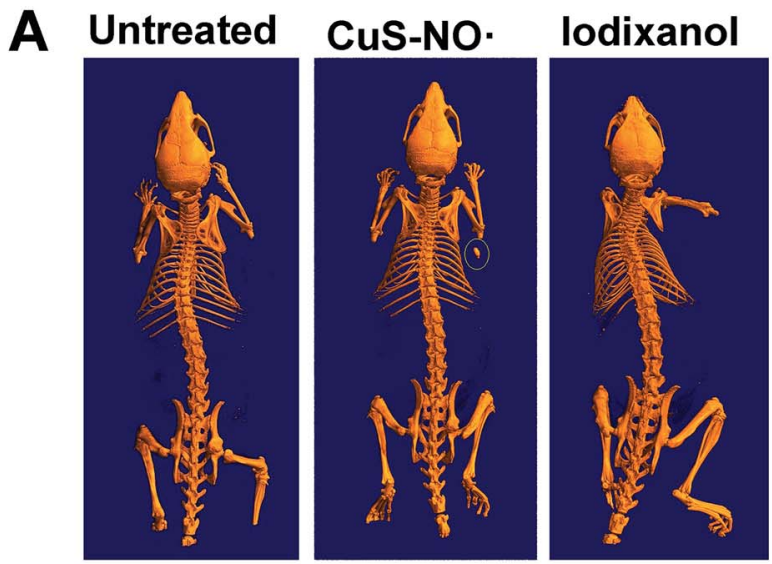

\section{B $30 \mathrm{~min}$}

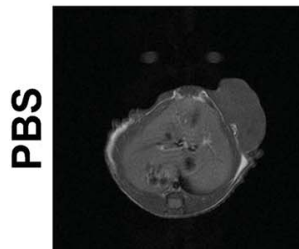

\section{$120 \mathrm{~min}$}

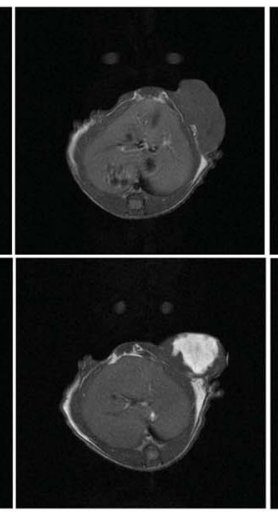

\section{$300 \mathrm{~min}$}

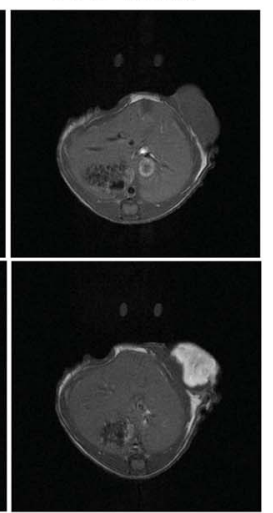

Fig. 3 (A) In vivo CT imaging of HeLa-xenograft-bearing mice intratumorally injected with nothing, CuS-NO* NPs or iodixanol. The tumor is marked by the yellow circle. (B) In vivo $\mathrm{T}_{1}$-weighted MRI imaging of HeLa-xenograft-bearing mice intratumorally injected with CuS-NO*NPs.

a $1177 \mathrm{~nm}$ laser to assess the photothermal effect of CuS-NO NPs in vivo. As displayed in Fig. 4A, after 10 min 1177 nm NIR irradiation $\left(0.5 \mathrm{~W} \mathrm{~cm}^{-2}\right)$ at $1 \mathrm{~h}$ after injection, there was no remarkable temperature elevation of tumor in the PBS group. In contrast, the temperature elevation of the tumor in the CuS-NO` NP group exhibited rapid and significant increase, implying CuS-NO` NPs could play an important role in NIR-II laser-responsive PTT treatment. As depicted in the temperature profiles of HeLa-tumour-bearing mice (Fig. 4B), both the PBS group and CuS-NO` NP group exhibited temperature increase as a function of irradiation time. However, the temperature of tumor sites in the PBS group rose slowly and reached $34.5{ }^{\circ} \mathrm{C}$ at $10 \mathrm{~min}$ after laser irradiation, suggesting that PBS could not be used for NIR-II laser-responsive PTT treatment. In contrast, the temperature of tumor sites in the CuS-NO ${ }^{\circ} \mathrm{NP}$ group increased rapidly and reached $55.2^{\circ} \mathrm{C}$ at $10 \mathrm{~min}$ after laser irradiation. Meanwhile, the higher the CuS-NO ${ }^{*} \mathrm{NP}$ concentration, the shorter the time needed to reach $55{ }^{\circ} \mathrm{C}$ (Fig. 4B), which demonstrated the potential of CuS-NO ${ }^{\circ}$ NPs in NIR-II laser-responsive cancer treatment.

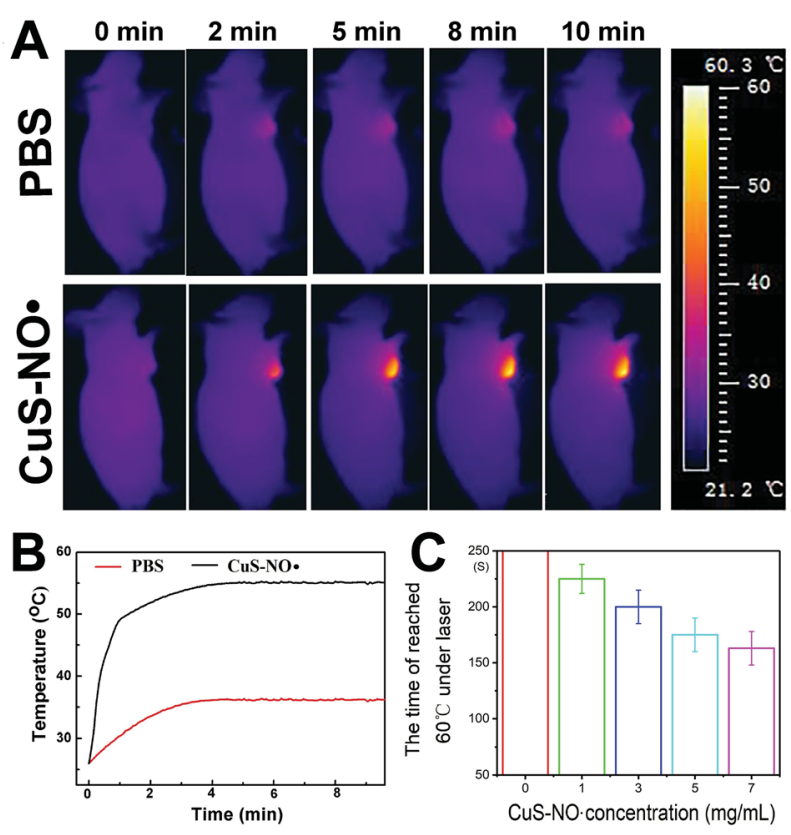

Fig. 4 (A) IR thermal images and (B) temperature profiles of HeLatumour-bearing mice intratumorally injected with CuS-NO• NPs (1 mg $\mathrm{mL}^{-1}$ ) or PBS and irradiated by a $1177 \mathrm{~nm}$ laser $\left(0.5 \mathrm{~W} \mathrm{~cm}{ }^{-2}\right)$ for $10 \mathrm{~min}$. (C) The time for different concentrations $(0,1.0,3.0,5.0$, and $7.0 \mathrm{mg}$ $\mathrm{mL}^{-1}$ ) to reach $55^{\circ} \mathrm{C}$.

\section{Flow cytometry and in vivo micro-PET effect}

Subsequently, $1177 \mathrm{~nm}$ laser light was selected, and the survival rate of HeLa cells incubated with different groups

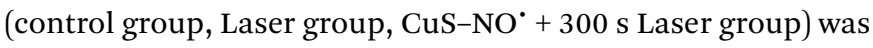
evaluated. As indicated in Fig. 5A, the control group cell survival rate $(\mathrm{Q} 4)$ was $81.7 \%$. However, the survival rate of the cells in the CuS-NO +300 s Laser group was only $28.8 \%$, further demonstrating that NIR-II laser-responsive PTT rendered through $\mathrm{CuS}_{-\mathrm{NO}}{ }^{\bullet} \mathrm{NPs}$ was effective in killing cancer cells.

PET imaging with ${ }^{18} \mathrm{~F}$-MISO was employed to illustrate the tumor volume and analyze the hypoxia condition after NIR-II laser-responsive PTT treatment rendered by CuS-NO` NPs or just after intratumoral injection of saline. As exhibited in Fig. 5B, compared with the observations for the saline group, the tumor volumes in the CuS-NO ${ }^{*} \mathrm{NP}$ group greatly regressed after treatment. More importantly, the two groups displayed different hypoxia degrees. The hypoxia signal in four different layers of a tumor from a mouse in the CuS-NO ${ }^{*}$ NP group was barely detected after treatment (Fig. 5B, white circle). In contrast, four different layers of a tumor from a mouse in the saline group showed serious hypoxia degree. Hypoxic zone has been reported to be associated with malignancy degree of tumor, and a high hypoxia level represents high tumor malignancy condition..$^{10,18}$ As such, small range of hypoxic zone meant NIR-II laser-responsive PTT rendered by CuS-NO` NPs was effective for tumor treatment. 

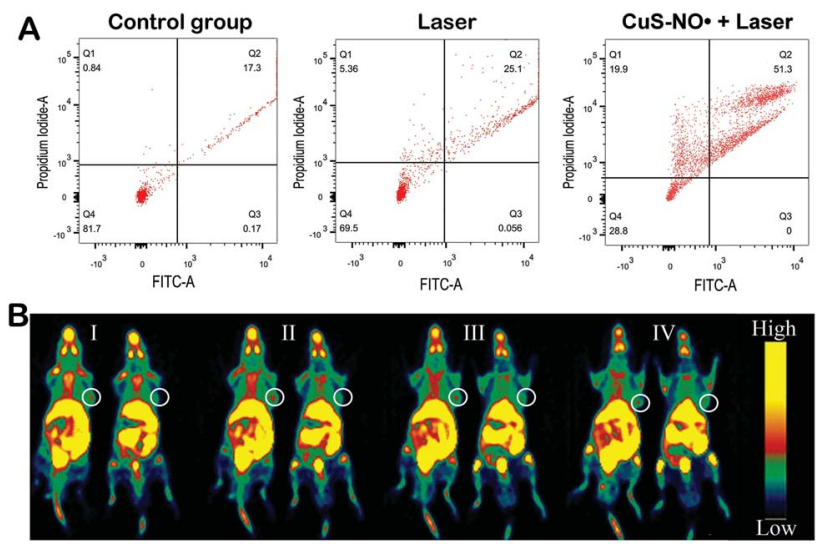

Fig. 5 (A) The cell flow diagrams after co-incubation with HeLa cells in different groups. (B) PET images of HeLa-tumour-bearing mice after NIR-II laser-responsive PTT treatment rendered by CuS-NO ${ }^{*}$ NPs or intratumoral injection of saline. I, II, III, and IV on right panels are four different layers of the tumor from a mouse in the CuS-NO• NP group. I, II, III, and IV on left panels are four different layers of the tumor from a mouse in the saline group. The tumors are marked with white circles.

\section{In vivo antitumor effect}

Having demonstrated that $\mathrm{CuS}^{-\mathrm{NO}}{ }^{*} \mathrm{NPs}$ could be used for CT/ MR imaging-guided NIR-II laser-responsive PTT treatment in vitro, their effects in vivo was next examined. HeLa-tumourbearing mice treated with $4 \mathrm{mg} \mathrm{kg}^{-1} \mathrm{CuS}-\mathrm{NO}^{\bullet} \mathrm{NPs}, 4 \mathrm{mg} \mathrm{kg} \mathrm{kg}^{-1}$ CuS-NO NPs with $1177 \mathrm{~nm}$ laser irradiation $\left(0.5 \mathrm{~W} \mathrm{~cm}^{-2}, 10\right.$ $\mathrm{min}$ ), and $4 \mathrm{mg} \mathrm{kg}^{-1}$ CuS NPs with $1177 \mathrm{~nm}$ laser irradiation $\left(0.5 \mathrm{~W} \mathrm{~cm}^{-2}, 10 \mathrm{~min}\right)$ were monitored for 25 days. Intratumoral injection of saline was used as the control. As can be seen in Fig. 6A, rapid growth of tumor tissues was observed in the saline group and CuS-NO ${ }^{*}$ NPs group, and tumor volumes on day 25 increased approximately 7.1- and 6.2-fold, respectively,
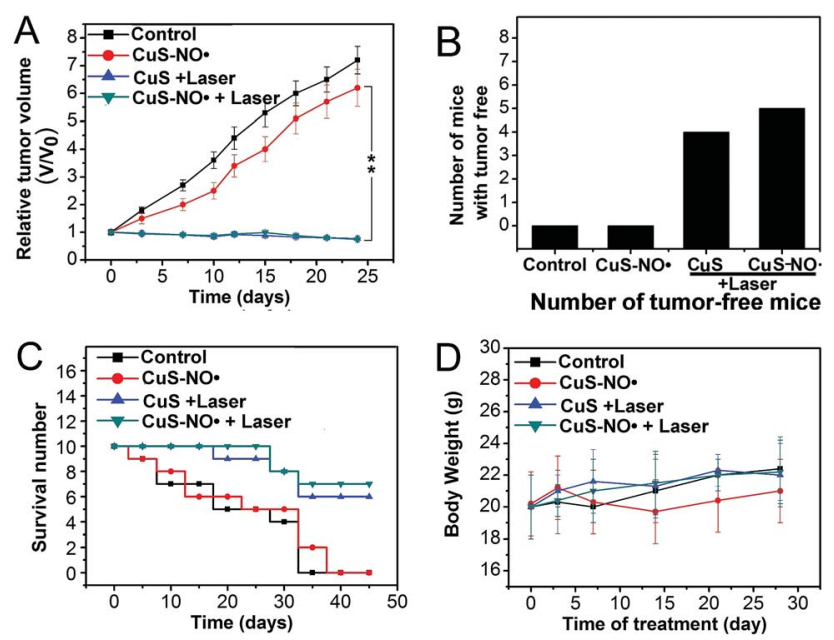

Fig. 6 (A) Tumor volumes, (B) number of tumor-free mice, (C) survival profiles, and (D) body weight of HeLa-tumor-bearing mice treated with $4 \mathrm{mg} \mathrm{kg}^{-1} \mathrm{CuS}-\mathrm{NO}{ }^{*} \mathrm{NPs}, 4 \mathrm{mg} \mathrm{kg}^{-1} \mathrm{CuS}-\mathrm{NO}{ }^{*}$ NPs with $1177 \mathrm{~nm}$ laser irradiation $\left(0.5 \mathrm{~W} \mathrm{~cm}^{-2}, 10 \mathrm{~min}\right)$, and $4 \mathrm{mg} \mathrm{kg}^{-1}$ CuS NPs with $1177 \mathrm{~nm}$ laser irradiation $\left(0.5 \mathrm{~W} \mathrm{~cm}^{-2}, 10 \mathrm{~min}\right)$. Each group has 10 mice, $* * p<0.01$. compared with those at day 0 . On the contrary, a dramatic decrease of tumor volume was observed in CuS-NO ${ }^{*}$ NPs and CuS NPs plus laser irradiation groups. Notably, as shown in Fig. 4, 5 and 6B tumor tissues from the mice completely disappeared in the CuS-NO ${ }^{*}$ NPs and CuS NPs plus laser irradiation groups, respectively, whereas no tumor tissue from the mice disappeared in the control and $\mathrm{CuS}^{-\mathrm{NO}^{*}}$ treated groups (Fig. 6B). The same trend was seen for the life spans (Fig. 6C). No mice were alive on day 35 and day 40 in the control group and $\mathrm{CuS}^{-\mathrm{NO}^{\circ}}$ group, respectively. In contrast, the mice in CuSNO` NPs and CuS NPs plus laser irradiation groups exhibited longer life spans; seven and six mice were alive at the end in the CuS-NO` NPs and CuS NPs plus laser irradiation groups, respectively.

Moreover, no clear changes occurred in the body weights of the mice throughout the experiment regardless of different treatments (Fig. 6D), confirming that $\mathrm{CuS}^{-\mathrm{NO}^{*}}$ had no acute toxicity.

\section{Pathological analysis and hematology assay}

The general conditions of tumor-bearing mice were monitored after treatment. There were no clear changes in the general conditions in mice after injection of CuS-NO ${ }^{*}$ NPs. The in vivo cytotoxicity to normal tissues was evaluated by H\&E staining in

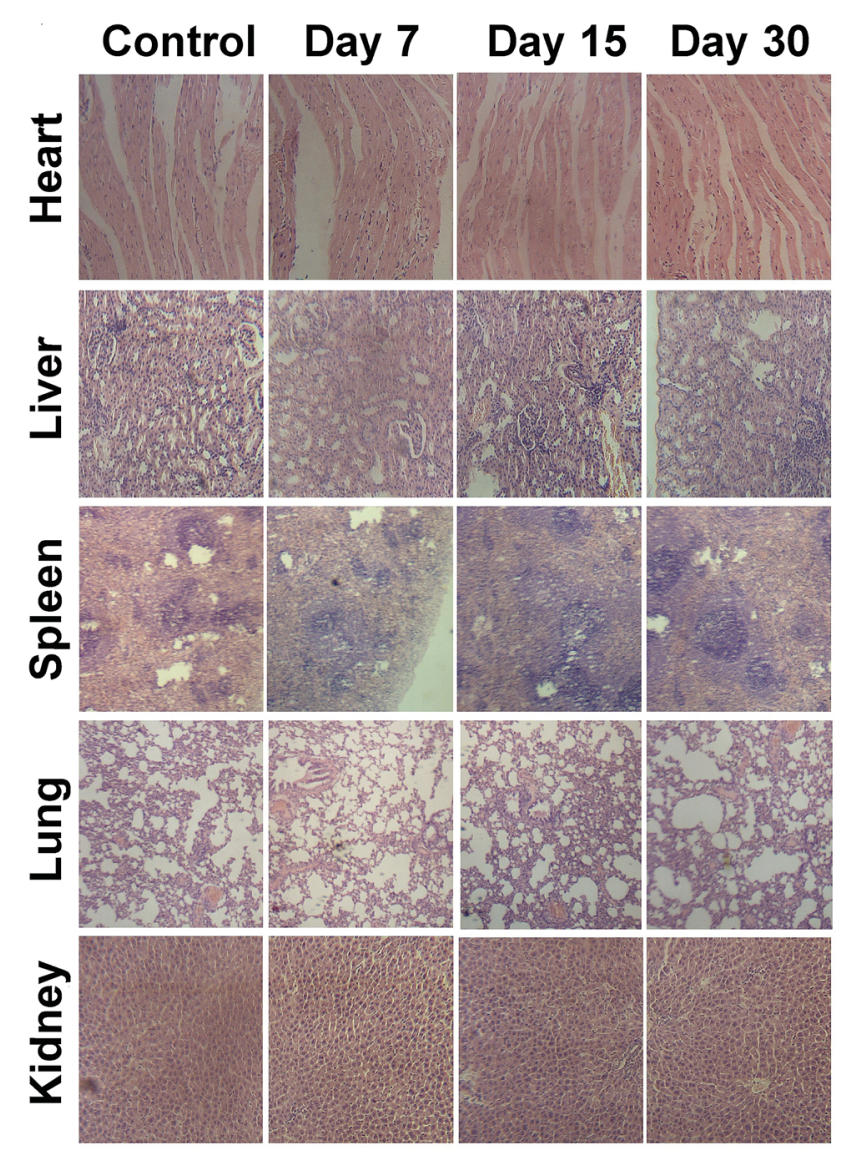

Fig. 7 H\&E staining images for different organs (kidney, lung, spleen, liver, and heart) from HeLa-tumor-bearing mice at 7, 15, and 30 days after NIR-II laser-responsive PTT treatment rendered by CuS-NO* NPs or intratumoral injection of saline. 
major organs. As Fig. 7 shows, compared with the observations for the control group, no apparent pathological changes, including necrosis, fibrosis, and hydropic degeneration, were observed in any organs isolated from CuS-NO ${ }^{*}$ NP-treated mice. These results confirmed the good biocompatibility of CuS-NO NPs.

Hematology and biochemical assays were conducted by collecting mouse blood on day 7, day 15 and day 30 after NIR-II laser-responsive PTT treatment rendered by $\mathrm{CuS}^{-\mathrm{NO}^{*}} \mathrm{NPs}$ or from untreated normal mice. Fig. 8A shows the levels of MON, PLT, EOS, LYM, BAS, and RBC; the values at 7, 15, and 30 days after NIR-II laser responsive PTT treatment rendered by CuSNO NPs were similar to those in normal mice, implying that negligible immune response had been aroused. The serum levels of ALT, AST, ALP, GLB, BUN, and Cr are connected with liver, kidney and spleen function. From Fig. 8B, no clear changes of ALT, AST, ALP, GLB, BUN, and Cr levels at 7, 15, and 30 days after NIR-II laser-responsive PTT treatment rendered by CuS-NO` NPs were detected compared with those in normal
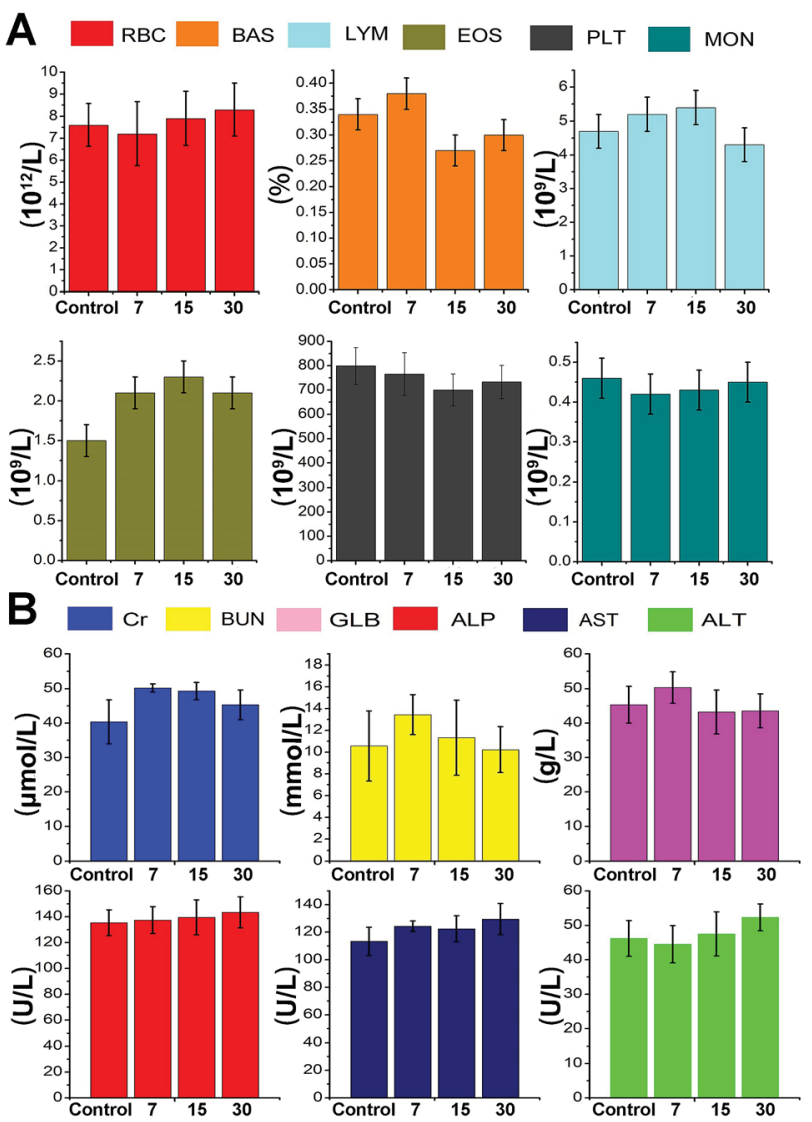

Fig. 8 (A) Serum bio-chemical study of peripheral blood monocytes (MON), platelets (PLT), eosinophils (EOS), lymphocytes (LYM), behavioural approach system (BAS), and red blood cell (RBC) levels at 7, 15, and 30 days after NIR-II laser-responsive PTT treatment rendered by CuS-NO NPs or intratumoral injection of saline. (B) The hematology assay of alanine aminotransferase (ALT), serum globulin (GLB), alkaline phosphatase (ALP), aminotransferase (AST), creatinine $(\mathrm{Cr})$, and blood urea nitrogen (BUN) levels at 7, 15, and 30 days after NIR-II laserresponsive PTT treatment rendered by $\mathrm{CuS}-\mathrm{NO}{ }^{*}$ NPs or intratumoral injection of saline. mice, indicating that the NIR-II laser-responsive PTT treatment rendered by CuS-NO ${ }^{*}$ NPs did not damage the liver, kidney and spleen functions. Collectively, these results demonstrated that CuS-NO` NPs are safe for in vivo bioapplications.

\section{Conclusions}

In summary, we tailor-made CuS-NO` NPs, which had a long wavelength absorbance and exhibited excellent therapeutic outcome in the NIR-II region. Meanwhile, negligible damage to normal tissues was detected due to the low energy $1177 \mathrm{~nm}$ laser used in this system. Besides, both the NO ${ }^{*}$ radicals and CuS nanoparticles were stable and separately contributed to enhanced CT and $\mathrm{T}_{1}$-weighted MR imaging, respectively. In this system, CuS-NO` NPs were simultaneously achieved for MRI and CT dual-modal imaging, successfully compensating for the low spatial resolution rendered by single CT imaging and negative response to calcification rendered by single $\mathrm{MR}$ imaging. Finally, CuS-NO` NPs for CT/MR dual-modal imagingguided tumor treatment may provide a new path for the clinical applications in the treatment of cancer.

\section{Conflicts of interest}

The authors report no conflicts of interest in this work.

\section{Acknowledgements}

This study was supported by Medical Science and Technology Development Foundation, Nanjing Department of Health (ZKX17032) and key medical subjects of Jiangsu Province (2016).

\section{Notes and references}

1 J. A. Joyce and J. W. Pollard, Nat. Rev. Cancer, 2009, 9, 239252.

2 D. F. Quail and J. A. Joyce, Nat. Med., 2013, 19, 1423-1437.

3 R. Mathew, V. Karantza-Wadsworth and E. White, Nat. Rev. Cancer, 2007, 11, 961-967.

4 J. Folkman, Semin. Cancer Biol., 2003, 13, 159-167.

5 K. Wang, X. Wu, J. Wang and J. Huang, Int. J. Nanomed., 2013, 8, 899-908.

6 D. P. Dearnaley, V. S. Khoo, A. R. Norman, L. Meyer, A. Nahum, D. Tait, J. Yarnold and A. Horwich, Lancet, 1999, 353, 267-272.

7 R. L. G. Lecaros, L. Huang, T. C. Lee and Y. C. Hsu, Mol. Ther., 2016, 24, 106-116.

8 C. Zhang, X. Cheng, M. Chen, J. Sheng, J. Ren, Z. Jiang, J. Cai and Y. Hu, Colloids Surf., B, 2017, 160, 345-354.

9 Y. Y. He, Y. Z. Shen, S. Q. Zhou, Y. H. Wu, Z. W. Yuan, C. Wei, L. J. Gui, Y. S. Chen, Y. Q. Gua and H. Y. Chen, RSC Adv., 2018, 8, 14268-14279.

10 C. Zhang, J. Ren, J. Hua, L. Xia, J. He, D. Huo and Y. Hu, ACS Appl. Mater. Interfaces, 2017, 10, 1132-1146. 
11 S. Zhao, Y. Tian, W. F. Liu, Y. Y. Su, Y. L. Zhang, Z. G. Teng, Y. Zhao, S. J. Wang, G. M. Lu and Z. H. Yu, RSC Adv., 2018, 8, 9023-9030.

12 A. X. Zheng, X. L. Zhang, Y. B. Huang, Z. X. Cai, X. L. Liu and J. F. Liu, $R S C A d v ., 2018$, 8, 6781-6788.

13 Z. Liu, J. Liu, R. Wang, Y. Du, J. Ren and X. Qu, Biomaterials, 2015, 56, 206-218.

14 F. P. Shu, D. J. Lv, X. L. Song, B. Huang, C. Wang, Y. Z. Yu and S. C. Zhao, RSC Adv., 2018, 8, 6581-6589.

15 L. Liang, S. W. Peng, Z. W. Yuan, C. Wei, Y. Y. He, J. R. Zheng, Y. Q. Gu and H. Y. Chen, RSC Adv., 2018, 8, 6013-6026.

16 R. M. Yang, C. P. Fu, J. Z. Fang, X. D. Xu, X. H. Wei, W. J. Tang, X. Q. Jiang and L. M. Zhang, Int. J. Nanomed., 2017, 12, 197-206.

17 R. K. Thapa, J. Y. Choi, B. K. Poudel, H. G. Choi, C. S. Yong and J. O. Kim, Int. J. Nanomed., 2016, 11, 2799-2813.

18 P. Zhao, S. Ren, Y. Liu, W. Huang, C. Zhang and J. He, ACS Appl. Mater. Interfaces, 2018, 10, 3405-3413.

19 C. Zhang, J. Ren, Y. Yang, D. Wang, J. He, D. Huo and Y. Hu, RSC Adv., 2016, 6, 113919-113923.

20 S. Ren, X. Cheng, M. Chen, C. Liu, P. Zhao, W. Huang, J. He, Z. Zhou and L. Miao, ACS Appl. Mater. Interfaces, 2017, 9, 31509-31518.

21 T. Cantu, K. Walsh, V. P. Pattani, A. J. Moy, J. W. Tunnell, J. A. Irvin and T. Betancourt, Int. J. Nanomed., 2017, 12, 615-632.

22 Z. Wang, Z. Chen, Z. Liu, P. Shi, K. Dong, E. Ju, J. Ren and X. Qu, Biomaterials, 2014, 35, 9678-9688.

23 S. Y. Madani, F. Shabani, M. V. Dwek and A. M. Seifalian, Int. J. Nanomed., 2013, 8, 941-950.

24 K. Dong, Z. Liu, J. Liu, S. Huang, Z. Li, Q. Yuan, J. Ren and X. Qu, Nanoscale, 2014, 6, 2211-2217.

25 Z. Liu, X. Liu, X. Ran, E. Ju, J. Ren and X. Qu, Biomaterials, 2015, 69, 56-64.

26 Z. Liu, J. Liu, R. Wang, Y. Du, J. Ren and X. Qu, Biomaterials, 2015, 56, 206-218.
27 A. F. Zedan, S. Moussa, J. Terner, G. Atkinson and M. S. ElShall, ACS Nano, 2013, 7, 627-636.

28 H. Yuan, A. M. Fales and T. Vodinh, J. Am. Chem. Soc., 2012, 134, 11358-11361.

29 M. Li, X. Yang, J. Ren, K. Qu and X. Qu, Adv. Mater., 2012, 24, 1722-1728.

30 L. Prodi, E. Rampazzo, F. Rastrelli, A. Speghini and N. Zaccheroni, Chem. Soc. Rev., 2015, 44, 4922-4952.

31 X. Ding, H. L. Chi, M. Zhang, R. Huang, C. Li, H. Shen, M. Liu, Y. Zou, N. Gao and Z. Zhang, J. Am. Chem. Soc., 2014, 136, 15684-15693.

32 X. J. Liu, Q. L. Ren, F. F. Fu, R. J. Zou, Q. Wang, G. B. Xin, Z. Y. Xiao, X. J. Huang, Q. Liu and J. Q. Hu, Dalton Trans., 2015, 44, 10343-10351.

33 Z. Meng, F. Wei, R. Wang, M. Xia, Z. Chen, H. Wang and M. Zhu, Adv. Mater., 2016, 28, 245-253.

34 L. Zhang, S. Gao, F. Zhang, K. Yang, Q. Ma and L. Zhu, ACS Nano, 2014, 8, 12250-12258.

35 A. Rajca, Y. Wang, M. Boska, J. T. Paletta, A. Olankitwanit, M. A. Swanson, D. G. Mitchell, S. S. Eaton, G. R. Eaton and S. Rajca, J. Am. Chem. Soc., 2012, 134, 15724-15727.

36 F. Hyodo, K. I. Matsumoto, A. Matsumoto, J. B. Mitchell and M. C. Krishna, Cancer Res., 2006, 66, 9921-9928.

37 G. Audran, L. Bosco, P. Brémond, J. M. Franconi, N. Koonjoo, S. R. A. Marque, P. Massot, P. Mellet, E. Parzy and E. Thiaudière, Angew. Chem., Int. Ed., 2015, 54, 13379-13384.

38 J. N. Liu, W. B. Bu and J. L. Shi, Chem. Rev., 2017, 117, 61606224.

39 L. Lin, X. Li, Y. Yang, L. Jing, X. Yue, X. Chen and Z. Dai, Curr. Drug Delivery, 2017, 14, 334-341.

40 C. Peng, L. F. Zheng, Q. Chen, Q. M. Shen, R. Guo, H. Wang, X. Y. Cao, G. X. Zhang and X.-Y. Shi, Biomaterials, 2012, 33, 1107-1119.

41 P. Moitra, K. Kumar, P. Kondaiah and S. Bhattacharya, Angew. Chem., 2014, 126, 1131-1135.

42 S. K. Misra, P. Moitra, P. Kondaiah and S. Bhattacharya, Colloids Surf., B, 2016, 142, 130-140. 